\title{
Expression of extracellular matrix proteins in cervical squamous cell carcinoma-a clinicopathological study
}

Iris Goldberg, Ben Davidson, Liat Lerner-Geva, Walter H Gotlieb, Gilad Ben-Baruch, Ilya Novikov, Juri Kopolovic

\begin{abstract}
Aim-To evaluate the intracellular and peritumoral expression of matrix proteins in squamous cell carcinoma of the uterine cervix using immunohistochemistry. Methods-71 squamous cell carcinomas and 10 controls were stained for laminin, fibronectin, and collagen IV. Cytoplasmic staining in tumour cells and peritumoral deposition of matrix proteins were evaluated. The association between staining results and patient age, tumour stage, histological grade, and survival was studied. Results-Positive cytoplasmic staining for laminin, fibronectin, and collagen IV was observed in $17(23.9 \%), 27(38 \%)$, and 10 (14.1\%) cases, respectively. Staining for laminin was most pronounced in the invasive front of tumour islands, while for fibronectin and collagen IV it appeared to be diffuse. Peritumoral staining for laminin and collagen IV was detected in 12 cases $(\mathbf{1 6 . 9 \% )}$. Early stage (Ia1-Ia2) tumours were uniformly negative for all three proteins. Cytoplasmic staining for laminin correlated with positive staining for fibronectin and collagen IV, and with the presence of a peritumoral deposition of collagen IV and laminin. There was no correlation with any of the three markers between staining results and patient age, stage, grade, or survival.
\end{abstract}

Conclusions-Expression of extracellular matrix proteins in some cervical squamous cell carcinomas might reflect the enhanced ability of these tumours to modify the peritumoral stroma. This ability seems to be absent in early stage tumours. The correlation between intracytoplasmic and peritumoral expression of matrix proteins supports the evidence of their synthesis by tumour cells. However, this property did not correlate with disease outcome in this study.

(f Clin Pathol 1998;51:781-785)

Keywords: collagen IV; laminin; fibronectin; uterine cervix; squamous cell carcinoma

The ability of neoplastic cells to invade the peritumoral stroma and subsequently metastasise depends on several factors. These include loss of adhesion, degradation of the underlying basement membrane, and complex interactions of cellular adhesion molecules with extracellular matrix components. ${ }^{1-9}$ Modification of extracellular matrix by neoplastic cells, medi- ated through the degradation, synthesis, and secretion of its components, appears to play a central role in tumour spread.

Several studies evaluated the expression of matrix proteins (for example, laminin, fibronectin, collagen IV) in carcinomas. Fibronectins, a family of high molecular weight adhesive glycoproteins, have been demonstrated immunohistochemically in breast ${ }^{10}$ and colorectal ${ }^{11}$ cancer cells. Laminins consist of a family of nine high molecular weight glycoproteins composed of varying $\alpha, \beta$, and $\gamma$ chains. $^{9}{ }^{12} 13$ Cytoplasmic/membranous expression of laminin has been shown in various carcinomas using immunohistochemistry, ${ }^{14-21}$ and laminin mRNA was detected in several epithelial tumours. ${ }^{17}{ }^{22-25}$ Laminin and fibronectin secretion and matrix deposition were observed in one study of cervical squamous cell carcinoma cell lines. ${ }^{22}$

Collagen IV (C IV), an amorphous collagen composed of $\alpha 1$ and $\alpha 2$ chains forming a triple helix, is normally present in all basement membranes. C IV mRNA has been detected in breast and salivary gland carcinomas. ${ }^{23} 24$

An association between matrix protein expression and histological and clinical variables has been observed for laminins and fibronectins. Indeed, laminin expression was noted in the invasive front of various carcinomas, ${ }^{17} 1921$ suggesting an association with tumour dissemination. In addition, altered expression of laminins and fibronectins, using immunohistochemical analysis, was associated with tumour grade and metastatic spread in salivary gland ${ }^{15}$ and oral cavity ${ }^{20}$ carcinomas. Expression of oncofetal (but not normal) fibronectin correlated with advanced stage and poor survival in colorectal carcinoma. ${ }^{11}$ Similar findings were not reported for C IV.

In this study we evaluated the intracellular expression of fibronectins, laminins, and C IV expression in 71 squamous cell carcinomas of the uterine cervix using immunohistochemistry. The peritumoral presence of laminins and C IV, as well as stromal staining for fibronectins, were also evaluated. We analysed the immunohistochemistry results in relation to patient age, tumour grade, clinical stage, and survival.

\section{Methods}

CLINICAL DATA

Seventy one patients with squamous cell carcinoma of the uterine cervix were treated and followed in the division of gynaecological
Accepted for publication 19 May 1998 
Table 1 Case distribution according to FIGO stage and survival

\begin{tabular}{lrccll}
\hline Stage & \multicolumn{1}{c}{$n$} & DOD & NED & REC & LFU \\
\hline IA1-2 & 8 & 0 & 8 & 0 & 0 \\
IB & 38 & 5 & 32 & 0 & 1 \\
II & 4 & 1 & 3 & 0 & 0 \\
III & 18 & 7 & 5 & 2 & 4 \\
IV & 3 & 3 & 0 & 0 & 0 \\
Total & 71 & 16 & 48 & 2 & 5 \\
\hline
\end{tabular}

DOD, died of disease; LFU, lost to follow up; NED, no evidence of disease; REC, recurrence of disease.

Table 2 Case distribution according to histological grade and survival

\begin{tabular}{lrrrll}
\hline Grade & $n$ & DOD & NED & REC & LFU \\
\hline 1 & 5 & 1 & 3 & - & 1 \\
2 & 30 & 3 & 24 & 1 & 2 \\
3 & 26 & 11 & 12 & 1 & 2 \\
$0 \dagger$ & 10 & 1 & 9 & - & - \\
Total & 71 & 16 & 48 & 2 & 5 \\
\hline
\end{tabular}

DOD, died of disease; LFU, lost for follow up; NED, no evidence of disease; REC, recurrence of disease.

†In 10 cases grade could not be assessed owing to scantiness of biopsy material or superficial invasion.

oncology at Sheba Medical Centre between 1984 and 1996. Follow up period ranged from one to 13 years (mean 3.4 years). The surgical material consisted of 32 hysterectomy specimens and 39 biopsy specimens. Ten normal cervices from hysterectomy specimens removed for leiomyomas were chosen as controls. Histological diagnosis of carcinoma was made according to FIGO criteria. ${ }^{26}$

Follow up surveillance included physical examination and cervical or vaginal cytology according to established clinical practice guidelines. Patients underwent yearly chest $x$ ray. Computed tomography and intravenous pyelography were performed as clinically indicated.

\section{ANTIBODIES}

Primary antibodies used in immunohistochemical staining were: monoclonal antilaminin LAM-89 (Sigma); monoclonal anticollagen type IV LH 7.2 (Sigma); and monoclonal anti-fibronectin 2232 MFIB (Euro-Diagnostica).

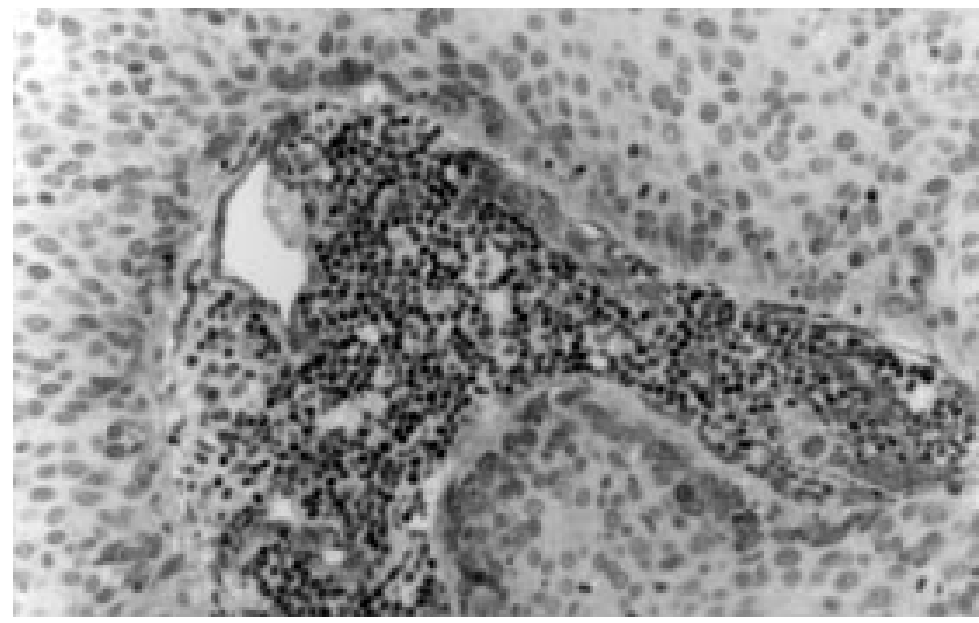

Figure 1 Laminin positivity in tumour cells facing an inflammatory infiltrate $(\times 49)$.

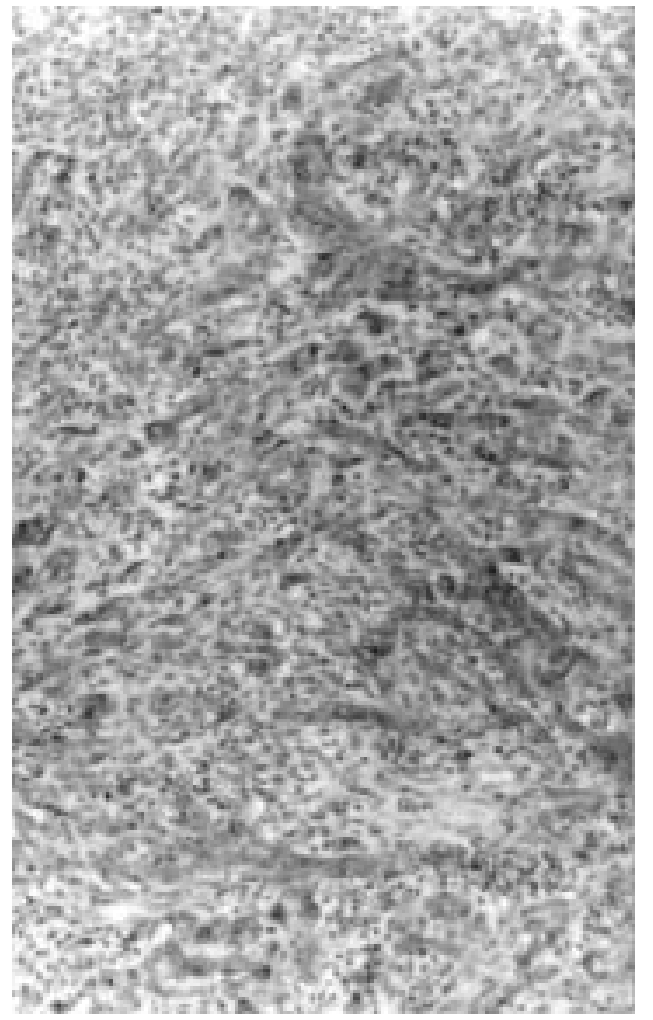

Figure 2 Fibronectin positive tumour cells (×39).

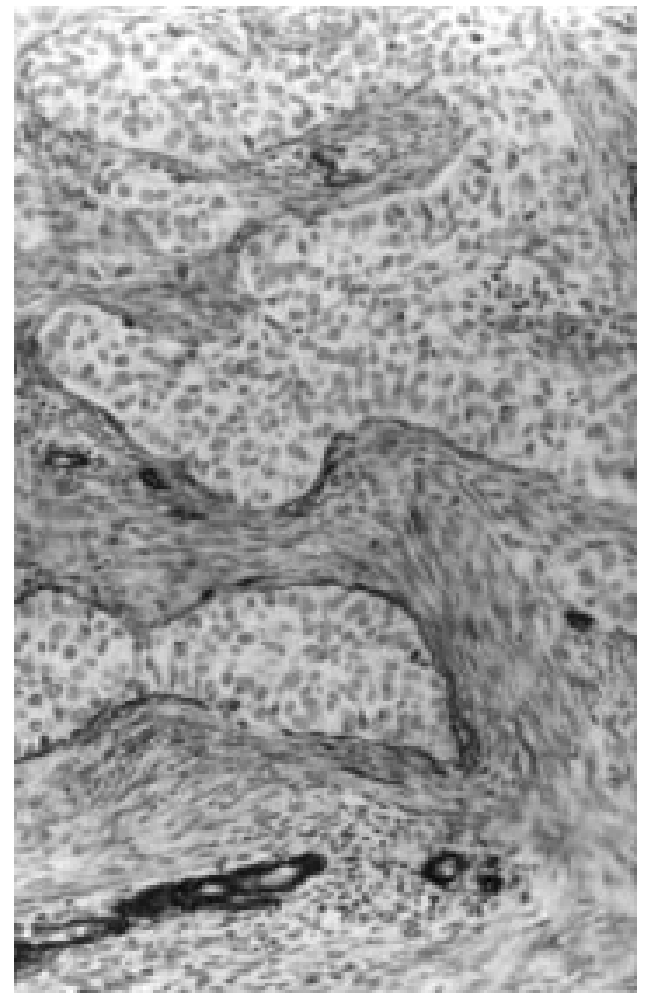

Figure 3 Fibronectin staining of peritumoral stroma. Tumour cells are negative $(\times 25)$.

IMMUNOHISTOCHEMICAL ANALYSIS

Immunohistochemical staining with the above antibodies was performed in all cases studied. Formalin fixed paraffin embedded sections, 4 microns thick, were thaw mounted onto Fisherbrand super frost/plus slides. After air drying at $37^{\circ} \mathrm{C}$ for 16 hours and incubation for 


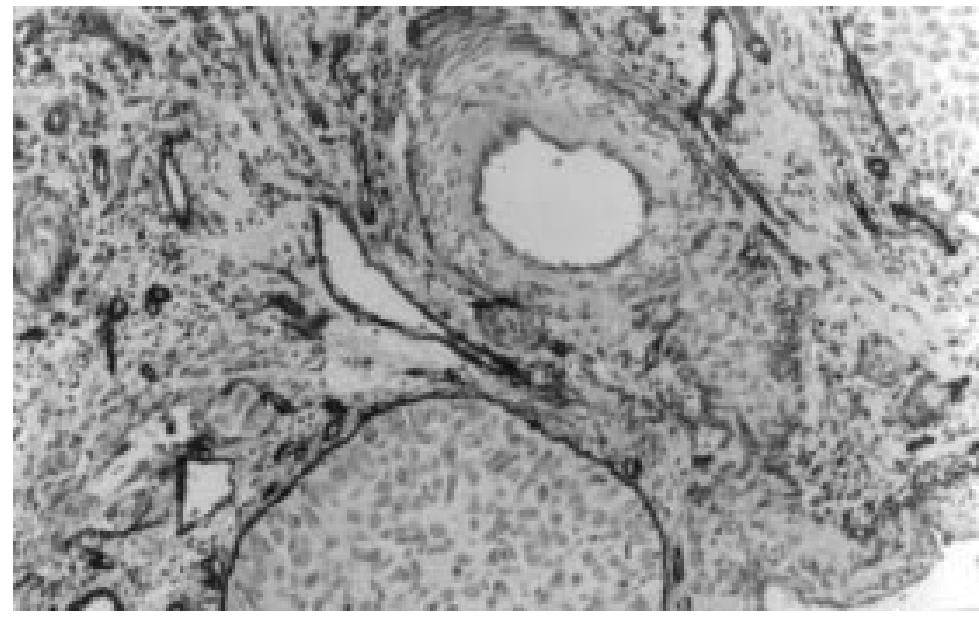

Figure 4 Peritumoral staining of tumour islands and staining of vascular basement membranes by anti-collagen IV $(\times 24)$.

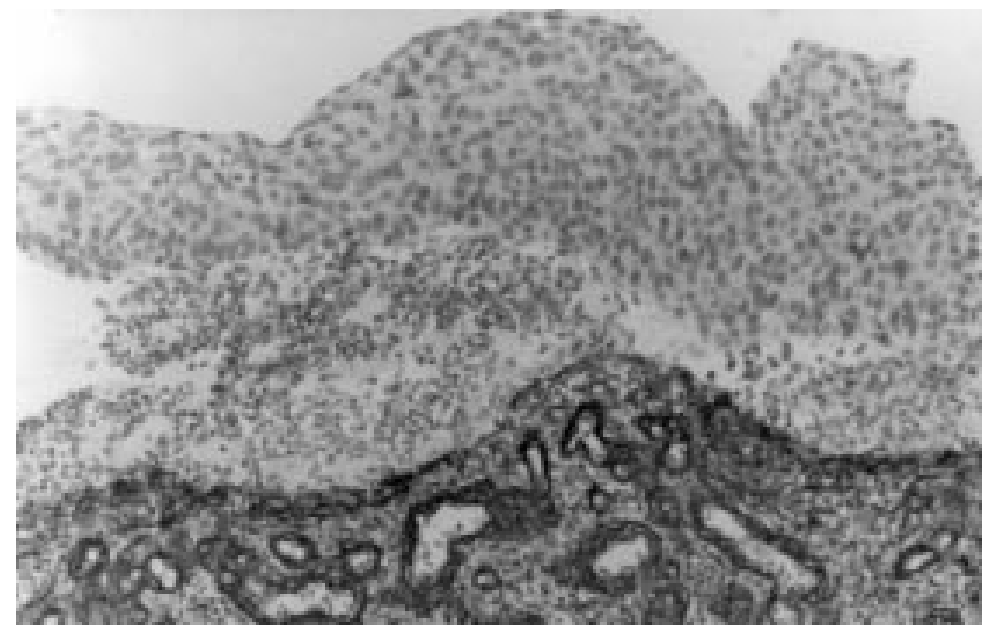

Figure 5 Staining of vessels by anti-collagen IV. Peritumoral staining was absent $(\times 24)$.

30 minutes at $60^{\circ} \mathrm{C}$, slides were deparaffinised, rehydrated, and separated into two groups for antigen exposure. The slides for fibronectin staining were placed in $10 \mathrm{mM}$ citrate buffer $(\mathrm{pH}=6.0)$ and heated twice for five minutes in a $750 \mathrm{~W}$ microwave oven. After each five minute interval the buffer was replenished and the oven was reactivated for a further five minutes. At the end of two cycles, slides were allowed to cool at room temperature for 10 minutes. The slides were rinsed for five minutes in Tris buffered saline (TBS; 0.05 $\mathrm{M}$ Tris/ $\mathrm{HCl}, 0.1 \mathrm{M} \mathrm{NaCl}, \mathrm{pH}=7.6$ ) containing $0.1 \%$ bovine serum albumin (BSA) and $0.05 \%$ Tween 20 (BSA-TBS-Tween). Slides for laminin and collagen IV staining were placed in $0.01 \mathrm{~N} \mathrm{HCl}$ with $0.4 \%$ pepsin for 30 minutes at $37^{\circ} \mathrm{C}$ for proteolytic digestion. To reduce background signals, all slides were incubated at room temperature with $10 \%$ non-immune goat serum for 15 minutes, followed by CAS block (Zymed Laboratories) for 30 minutes. For laminin and collagen IV staining, the antibodies were applied to the slides and incubated at room temperature for 30 minutes. Fibronectin staining was performed at $4^{\circ} \mathrm{C}$ for 16 hours. Staining was performed with labelled avidin-biotin (LAB). ${ }^{27} 28$
The presence of cytoplasmic staining for laminins, fibronectins, and C IV was evaluated in neoplastic cells and controls. Staining of more than $5 \%$ of tumour cells was interpreted as positive. As staining for fibronectins was expected in stromal cells, negative staining of inflammatory cells was used to exclude false positive non-specific staining. In addition, the presence of linear peritumoral staining for C IV and laminins, and stromal staining for fibronectins, were evaluated. Staining of vascular basement membranes was used as internal control. Staining of the subepithelial and vascular basement membranes was evaluated in controls.

\section{STATISTICAL ANALYSIS}

Statistical analysis was performed using the SPSS-PC package (version 6.1, SPSS, Chicago, 1996). A probability (p) value of $<0.05$ was considered statistically significant. Continuous variables were compared using Student's $t$ test analysis. Non-parametric analyses (Mann-Whitney test) and the $\chi^{2}$ test were used for categorical variable comparisons and trends. Survival analysis was performed using the Cox proportional regression model.

\section{Results}

The patients' mean age at the time of diagnosis was 47.8 years (range 27 to 83 ).

Sixteen patients died of disease, two suffered disease recurrence, and 48 remained free of disease. Five patients were lost to follow up. Case distribution according to tumour stage and grade are detailed in tables 1 and 2. For statistical purposes, tumours were grouped in three categories: stages Ia1-Ia2 (eight patients), stages Ib-II (42 patients), and stages III-IV (21 patients).

Positive cytoplasmic staining for laminins was found in 17 cases $(23.9 \%)$. Staining was most pronounced in the invasive front of tumour islands (fig 1). Positive cytoplasmic staining for fibronectins was detected in 27 cases (38\%) (fig 2). In contrast to the pattern of staining for laminins, staining for fibronectins was diffuse, showing similar intensity in tumour cells in both the invasive front and the central areas of tumour islands. The peritumoral stroma was positive for fibronectins in all cases (fig 3). Staining for fibronectins and laminins was intense in all positive cases, showing a homogeneous pattern for laminins and a predominantly granular pattern for fibronectins.

Weak cytoplasmic staining for C IV was detected in 10 cases $(14.1 \%)$. Linear peritumoral staining for C IV and laminins was detected in 12 cases $(16.9 \%$ ) (fig 4$)$. Vascular basement membranes in the vicinity of tumour islands stained positively with all three antibodies in all cases (fig 5). Cervical epithelium in control cases stained negatively with all three markers, while vascular and subepithelial basement membranes stained positively.

The distribution of staining results according to stage and grade is shown in tables 3 and 4 , respectively. 
Table 3 Distribution of cases positive for laminin, fibronectin, and collagen IV in relation to FIGO stage

\begin{tabular}{lrrrcr}
\hline Stage & \multicolumn{1}{c}{$n$} & $L N$ & $F N$ & $C I V$ & $P T$ \\
\hline IA1-2 & 8 & 0 & 0 & 0 & 1 \\
IB & 38 & 10 & 18 & 7 & 8 \\
II & 4 & 2 & 3 & 1 & 1 \\
III & 18 & 5 & 6 & 2 & 2 \\
IV & 3 & 0 & 0 & 0 & 0 \\
Total & 71 & 17 & 27 & 10 & 12
\end{tabular}

C IV, collagen IV; FN, fibronectin; LN, laminin; PT, peritumoral deposition of C IV and LN.

Table 4 Distribution of positive cases for laminin, fibronectin, and collagen IV in relation to histological grade

\begin{tabular}{lcrrcc}
\hline Grade & $n$ & $L N$ & $F N$ & $C I V$ & $B M$ \\
\hline 1 & 5 & 1 & 3 & 1 & 0 \\
2 & 30 & 9 & 12 & 6 & 6 \\
3 & 26 & 5 & 10 & 2 & 5 \\
$0 \dagger$ & 10 & 2 & 2 & 1 & 1 \\
Total & 71 & 17 & 27 & 10 & 12 \\
\hline
\end{tabular}

CIV, collagen IV; FN, fibronectin; LN, laminin; PT, peritumoral deposition of CIV and LN.

$\dagger$ In 10 cases grade could not be assessed due to scantiness of biopsy material or superficial invasion.

Table 5 Multivariate analysis of factors related to survival in a Cox regression model

\begin{tabular}{lrllll}
\hline & $\begin{array}{l}\text { Regression } \\
\text { coefficient }\end{array}$ & $\begin{array}{l}\text { Standard } \\
\text { error }\end{array}$ & $\begin{array}{l}\chi^{2} \\
\text { Value }\end{array}$ & $\begin{array}{l}p \\
\text { Value }\end{array}$ & $\begin{array}{l}\text { Hazard } \\
\text { ratio }\end{array}$ \\
\hline Age (years) & -0.0010 & 0.0186 & 0.0030 & 0.9561 & 0.9990 \\
Grade & 0.4911 & 0.3742 & 1.9980 & 0.1575 & 1.6341 \\
Stage & 0.8022 & 0.2881 & 7.7413 & $0.0054^{\star}$ & 2.2305 \\
Laminin & 0.1193 & 0.6817 & 0.0302 & 0.8620 & 1.1267 \\
Fibronectin & -0.2155 & 0.7029 & 0.0968 & 0.7557 & 0.8061 \\
Collagen IV & -0.9494 & 1.2451 & 0.6421 & 0.4229 & 0.3870 \\
\hline * Statistically significant. & & & &
\end{tabular}

The association between staining results and patient age, tumour stage, histological grade, and survival was studied. Tumour cells in stage Ia1-Ia2 and stage IV were uniformly negative for all three markers. Positive cytoplasmic staining for laminins correlated with cytoplasmic staining for fibronectins and C IV, as well as with presence of peritumoral staining for laminins and C IV $(p=0.01)$. Survival was associated with clinical stage, but not with the staining results by all three markers (table 5).

\section{Discussion}

In this study, we evaluated the expression of extracellular matrix proteins in 71 uterine cervical squamous cell carcinomas using immunohistochemistry.

In agreement with previous studies, staining for laminin (laminins) was most pronounced at the invasive front of the tumour islands. The demonstration of laminin mRNA and protein expression in carcinomas ${ }^{17}{ }^{22-24}$ indicates synthesis of laminins by tumour cells. Laminin deposition in the vicinity of tumour islands, reported previously, ${ }^{14-20}$ was observed in two thirds of the laminin positive tumours. We found no association between laminin positivity and histological grade in our study. This finding differs from the observation of d'Ardenne et al in a study of oral cavity and salivary gland adenoid cystic carcinomas. ${ }^{15}$ However, only six tumours were analysed in that study.

In contrast to laminin, fibronectin positive tumours showed a diffuse staining pattern, independent of cell location. The granular staining pattern observed could be caused by the intracellular location of fibronectins in lysosomes, resulting from extracellular matrix degradation by tumour cells. Metalloproteinases, a family of zinc and calcium dependent proteolytic enzymes, play a central role in extracellular matrix degradation. Both gelatinases and stromelysins, two subgroups of metalloproteinases, are able to degrade fibronectins, and stromelysins degrade laminins as well. ${ }^{29}$ We have localised both gelatinases and stromelysins to cervical carcinoma cells (Davidson B, et al, unpublished data). On the other hand, the diffuse pattern of fibronectin staining may point to a less prominent role for fibronectin deposition in the process of invasion. The lack of correlation between tumour grade and fibronectin expression is in agreement with the findings in colorectal carcinoma. ${ }^{11}$

A weak cytoplasmic staining for C IV was noted in 10 cases. In seven of these, as well as in a further five cases, staining was associated with the presence of linear peritumoral staining for both laminins and C IV. Peritumoral deposition of laminins and C IV correlated with cytoplasmic expression of all three proteins. This correlation reflects the propensity of these tumours to participate in the modification of most matrix components.

No correlation was found between disease stage or survival and positive staining for any of the markers examined. However, invasive cervical carcinomas more commonly expressed matrix proteins than controls or cases of preinvasive cervical intraepithelial neoplasia (CIN) grades I, II, and III. ${ }^{30}$

The ability to invade does not necessarily confer on the cells the ability to metastasise. This might explain the lack of correlation between the expression of laminins, fibronectins, and C IV, and the presence of metastases or survival in our study. Controversial findings have been reported in colorectal and breast carcinoma. Fibronectin expression did not correlate with disease outcome in colorectal carcinoma, ${ }^{11}$ but absence of its expression predicted a worse outcome in breast carcinomas. ${ }^{10}$ In addition, laminin expression was associated with poor prognosis in a subgroup of breast carcinomas that lacked the laminin receptor. ${ }^{16}$

Interestingly, none of the stage Ia1-Ia2 tumours stained positively for laminins, fibronectins, or C IV, and in only one of eight cases could we identify deposition of peritumoral laminins and C IV. Early stage tumours therefore seem to have a lesser capacity to synthesise or degrade matrix proteins. Thus the ability to modify extracellular matrix through deposition of its constituents seems to characterise tumours that invade and that are able to spread locally, as opposed to their minimally invasive counterparts. This finding is further supported by those of Pyke et al for laminins in a study of 72 malignant tumours, including six carcinomas of uterine cervical origin. ${ }^{17}$ 
Notably, all three stage IV carcinomas that we studied were negative for all markers. Although this subgroup is small, one may reflect on whether tumours that are able to metastasise to distant organs downregulate matrix protein turnover as a result of decreased activity in local invasion.

In conclusion, based on the study of 71 uterine cervical squamous cell carcinomas, no correlation could be found between survival and laminin, fibronectin, or collagen IV expression. However, minimally invasive lesions and stage IV tumours did not express any of the proteins examined, in contrast to locally invasive tumours. In addition, a strong correlation was observed between the cytoplasmic expression of all three proteins, and between these cytoplasmic proteins and the peritumoral deposition of laminins and collagen IV. More detailed studies of specific laminin chains or fibronectin isoforms may shed some light on the role of these proteins in the invasive process of cervical neoplasia.

We thank Mrs Bartenstein for her dedicated work in the immunohistochemistry laboratory.

1 Bernstein LR, Liotta LA. Molecular mediators of interactions with extracellular matrix components in metastasis tions with extracellular matrix components in m

2 Tuszynski GP, Wang TN, Berger D. Adhesive proteins and the hematogenous spread of cancer. Acta Haematol 1997;97:29-39.

3 Albelda SM, Buck CA. Integrins and other cell adhesion molecules. FASEB f 1990;4:2868-80.

4 Albelda SM. Role of integrins and other cell adhesion molecules in tumor progression and metastasis. Lab Invest 1993;68:4-17.

5 Ruoslahti E. Fibronectin and its receptors. Annu Rer Biochem 1988;57:375-413.

6 Akiyama SK, Olden K, Yamada KM. Fibronectin and integrins in invasion and metastasis. Cancer Metastasis Rev 1995;14:173-89.

7 Juliano RL. The role of $\beta 1$ integrins in tumors. Semin Cancer Biol 1993;4:277-83.

8 Ruoslahti E, Filippo CC. Integrins and tumor cell dissemination. Cancer Cells 1989;1:119-26.

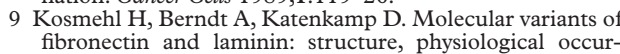
fibronectin and laminin: structure, physiological occur-
rence and histopathological aspects. Virchows Arch 1996; rence and histo

10 Takei H, Iino Y, Horiguchi J, et al. Immunohistochemical fibronectin staining pattern and prognosis in invasive breas carcinoma. Oncology 1995;52:106-11.

11 Inufusa $\mathrm{H}$, Nakamura $\mathrm{M}$, Adachi $\mathrm{T}$, et al. Localization of oncofetal and normal fibronectin in colorectal cancer. Correlation with histologic grade, liver metastasis, and prognosis. Cancer 1995;75:2802-8.
12 Kleinman HK, Cannon FB, Laurie JR, et al. Biological Keinman HK, Cannon FB, Laurie JR, et al. Biologi
activities of laminin. $f$ Cell Biochem 1985;27:317-25.

13 Tryggvason K. The laminin family. Curr Opin Cell Biol 1993;5:877-82.

14 Albrechtsen R, Nielsen M, Wewer U, et al. Basement membrane changes in breast cancer detected by immunohistochemical staining for laminin. Cancer Res 1981;41:507681 .

15 d'Ardenne AJ, Kirkpatrick P, Wells CA, et al. Laminin and fibronectin in adenoid cystic carcinoma. f Clin Pathol 1986;39:138-44.

16 Pellegrini R, Martignone S, Tagliabue E, et al. Prognostic significance of laminin production in relation with its receptor expression in human breast carcinomas. Breast Cancer Res Treat 1995;35:195-9.

17 Pyke C, Salo S, Ralfkiaer E, et al. Laminin-5 is a marker of invading cancer cells in some human carcinomas and is coexpressed with the receptor for urokinase plasminogen activator in budding cancer cells in colon adenocarcinomas. Cancer Res 1995;55:4132-9.

18 David L, Nesland JM, Holm R, et al. Expression of laminin, collagen IV, fibronectin, and type IV collagenases in gastric carcinoma. An immunohistochemical study of 87 patients. Cancer 1994;73:518-27.

19 Tani T, Karttunen T, Kiviluoto T, et al. $\alpha 6 \beta 4$ integrin and newly deposited laminin- 1 and laminin- 5 form the adhesion mechanism of gastric carcinoma. Continuous expression of laminins but not that of collagen VII is preserved in invasive parts of the carcinomas: implications for acquisition of the invading phenotype. Am $\mathcal{f}$ Pathol 1996;149:781-93.

20 Kainulainen $\mathrm{T}$, Autio-Harmainen $\mathrm{H}$, Oikarinen A, et al. Altered distribution and synthesis of laminin-5 (kalinin) in Altered distribution and synthesis of laminin-5 (kalinin) in oral lichen planus, epithelial dysplasias and
carcinomas. $\operatorname{Br} \mathcal{7}$ Dermatol 1997;136:331-6.

21 Pyke C, Romer J, Kallunki P, et al. The $\gamma 2$ chain of kalinin/ aminin 5 is preferentially expressed in invading malignant cells in human cancers. Am f Pathol 1994;145:782-91.

22 Auersperg N, Kruk PA, MacLaren IA, et al. Heterogeneous expression of keratin, involucrin and extracellular matrix among subpopulations of a poorly differentiated human cervical carcinoma: possible relationships to patterns of invasion. Cancer Res 1989;49:3007-14

23 Soini Y, Autio-Harmainen H. Synthesis and degradation of basement membranes in benign and malignant salivary glands tumors. A study by in situ hybridization. $\mathcal{F}$ Pathol 1993;170:291-6.

24 Soini Y, Hurskainen T, Hoyhtya M, et al. $72 \mathrm{kD}$ and $92 \mathrm{kD}$ type IV collagenase, type IV collagen, and laminin mRNA in breast cancer: a study by in situ hybridization. $\mathcal{F}$ Histochem Cytochem 1994;42:945-51.

25 Hao J, Yang Y, McDaniel KM, et al. Differential expression of laminin $5(\alpha 3 \beta 3 \gamma 2)$ by human malignant and normal prostate. Am f Pathol 1996;149:1341-9.

26 Rosai J. FIGO staging for carcinoma of the cervix uteri. In: Ackerman's surgical pathology, 8th ed, vol 2. St Louis: CV Mosby, 1996:1367.

27 Giorno R. A comparison of two immunoperoxidase staining methods based on the avidin-biotin interaction. Diagn Immunol 1984;2:161-6.

28 Guesdon JL, Ternynck T, Avrameas S. The use of avidin-biotin interaction in immunoenzymatic techniques. 7 Histochem Cytochem 1979;27:1131-9.

29 Aznavoorian S, Murphy AN, Stetler-Stevenson WG, et al. Molecular aspects of tumor cell invasion and metastasis. Cancer 1993;71:1368-83.

30 Davidson B, Goldberg I, Gotlieb WH, et al. Expression of matrix proteins in uterine cervical neoplasia. Immunohistochemical study. Eur 7 Obstet Gynecol Reprod Biol (in press). 